\title{
Numerus et Sapientia
}

\author{
"Veritatis et sapientiae pulchritudo... de toto \\ mundo ad se conversis, qui diligunt eam, omnibus \\ proxima est, omnibus sempiterna; nullo loco est, \\ nusquam deest; foris admonet, intus docet; cer- \\ nentes se commutat, a nullo in deterius commuta- \\ tur; nullus de illa judicat, nullus sine illa judicat \\ bene... Ab ipsa fiunt singulae (mentes) sapientes, \\ et non de ipsa, sed per ipsam de caeteris judices. \\ De Lib. arb. II, 14, 38, PL. 32, 1262.
}

Sumario: Número estético y número metafísico-Número radical y número ideal.-Número platónico y número cristiano.-iSíntesis de platonismo y cristianismo?-Ejemplo de Heidegger.-Número y dialéctica.-Reino objetivo, presente y apriorístico.-Número y sabiduría, a la luz de la Biblia y de la Filosofía.-Irradiación divina e inteligibilidad humana.-El número como primer principium quo del ser, para explicar la dialéctica.

Cuando se pretende aquilatar el sentido y el valor del número agustiniano, surgen no pocas indeterminaciones aparentes. $\mathrm{Y}$ en primer lugar, surge la ambigüedad fundamental, ya que no sabemos si considerar el número estéticamente o bien metafísicamente. En el primer caso, tendríamos una manifestación o revelación del ser, pero de un ser-valor que llamamos "bello" o "feo", y que se realiza a nivel consciente, como cxpresión de una esencia o forma. En el segundo caso, tendríamos una impresión o formación elemental del ser, antes de que sea expresado en una esencia: lo consideramos entonces como un valor-ser, que llamamos número, y que ponemos a nivel inconsciente para nosotros, motivo de demostración, no de percepción directa o inmediata. Acerca de ambos sentidos tiene muchos textos Agustín. Comenzó su carrera literaria escribiendo un diálogo "Sobre lo bello y sobre lo apto", que hoy se ha perdido. Creemos que Agustín tomó de Varrón y de la literatura latina cl término numerus, con sus derivados numerosus, numerositas, etc., como sinónimos de razonable, formado, bello, es decir, en sentido preferentemente estético. De ahí que haga tantas aplicaciones a la matemática, música, arquitectura y demás artes, como puede verse en el librito De Ordine. Los "números" son entonces explicados etimológicamente 
como "ritmo", esto es, como módulo, compás, correspondencia, igualdad, etc., esto es, como medidas o intervalos temporales o espaciales. Así, las columnas de un templo guardan simetría y ritmo espacial, ya que están colocadas a espacios iguales: del mismo modo, los movimientos de una bailarina, que danza, se van ajustando a un compás, es decir, a espacios temporales iguales y correspondientes: finalmente, en ese sentido la pronunciación científica de un verso latino es toda una lección de música, de numerosidad, como se ve en el librito De Música. Pero cuando Agustín aceptó el platonismo, comenzó a dar al término número un sentido más profundo, metafísico. Se trata aquí ya de un "ser" y no de un "parecer": pero se trata de un ser que no vemos, y que sólo podemos demostrar; no es un problema físico, aunque la física lo aproveche para sus especulaciones, sino que es un problema metafísico, totalmente filosófico.

Pero el platonismo implica una nueva indeterminación. En efecto, Agustín nos habla de un número radical, que viene a identificarse con la unidad radical, con el ser mismo, por así decirlo, antes de que ese ser tenga esencia alguna, ya que esta esencia será aplicada al ser según un "número" al que se tiene que ajustar para no exceder de sus límites. Pero habla asimismo de un número ideal, es decir, de una esencia perfecta, a cuya realización aspira el ser radical. Un niño, que nace, no es todavía nada, es un hombre "en potencia"; un "santo" que muere es un ideal humano. Entre ambos hay una vida dialéctica, un proceso de ejecución. Suponiendo pues que el número platónico, adoptado por Agustín significase una superación (cosa imposible, ya que el valor belleza es irreductible y autónomo) del estoicismo, quedaría ante nosotros una nueva indeterminación, siendo preciso escoger entre el número radical y el número ideal. En realidad estamos ya ante dos indeterminaciones a la vez.

Agustín no se contenta con ser platónico, sino que aspira a unir eì platonismo con el cristianismo. $\mathrm{Y}$ entonces surge ante nosotros una nueva indeterminación ${ }^{1}$, aunque en realidad el platonismo quede subordinado al cristianismo. Heidegger es un ejemplo moderno de esa indeterminación:

"A dirigir la vista a la "cura", y a mantenerla fija en ella, dentro de la analítica existenciaria del Dassein, le llevó al autor el intento de hacer

1 Confess. VII, 10, 16, PL. 32, 742-744. 
una exégesis de la antropología agustiniana, e. d. grecocristiana, en relación con los fundamentos radicales de la ontología de Aristóteles" 2.

¿Es posible una síntesis en tales condiciones? Heidegger utiliza a Agustín y a Aristóteles para una construcción moderna: la ontología tiene todavía vigencia y ha de ser reconstruída bajo la dirección de los griegos y de Agustín. Este ejemplo de Heidegger pone a la vista el problema sintético.

Eso mismo hemos pensado nosotros, al querer superar la indeterminación agustiniana de los dos sentidos del número. Vemos, en efecto, que el mismo Agustín relaciona el número con la sabiduría y aún llega a identificarlos, colocando de ese modo a la antropología como vía de acceso a la ontología, tal como lo hizo Heidegger, aunque su libro haya quedado sin terminar. Agustín y Heidegger toman al hombre como modelo: descubren en él una sapientia originaria, que no es una simple idea, sino también una energía o virtud capaz de llevarlo hasta su perfección ideal, siempre que no se oponga algún obstáculo; eso significa la tendencia del alma a unificar ${ }^{3}$. Pero en Agustín, esa tendencia, al mismo tiempo ejemplar o formal y ejecutiva u operativa, se descubre en todas las demás cosas del "Universo", hasta el punto de haber soñado construir una "filosofía de la unidad", que podría equivaler a la "enología" neoplatónica en el campo cristiano".

La "dialéctica" es pues el modo de existir del "Universo" agustiniano. Entre cada ser radical y su idea o unidad perfecta hay un movimiento, proceso o entelequia. Y la fuerza trófica que se esconde en el misterio de cada ser, y que mantiene ese ser en la existencia y lo hace caminar hacia la perfección, es, no un amor, como creía Max Scheler, o una idea, como creían Platón y Aristóteles, sino un Número, una Unidad, que participa al mismo tiempo de la Verdad y del Amor, al participar de la Unidad Primaria. Cada cosa de este mundo lleva impresa en el corazón esa unidad trina, que es al mismo tiempo modelo y energía, virtus et sapientia, ut unum aliquod perfectum fiat ${ }^{5}$. Tanto

2 M. Heidegger, Ser y Tiempo, México 1951, 229, nota 2.

3 De Ordine II, 18, 48, PL. 32, 1017.

4 Ibid. En Plotino se trata del Unum in nobis y del unum in rebus, que proviene de la emanación de todas las cosas a partir de Lo Uno, y que la razón puede descubrir, cuando entra en sí misma. En Agustín el problema sería diferente, desde eil momento en que parte de la creación cristiana.

5 Estos conceptos venían de Cicerón, del Estoicismo, pero esto no interesa de momento. Cfr. De Libero Arbitrio I, 6, 15, PL. 32, 1229. 
la verdad como el amor son funciones, instrumentos u órganos de esa unidad misteriosa, oculta en el fondo de cada ser, como la chispa o centella (Funkelein) de los místicos.

Agustín aprovecha desde el principio el concepto de Ley Eterna, que se copia o transcribe en el alma del hombre y se llama entonces Ley Natural, para explicar el fundamento de la sabiduría humana. La ley natural viene "impresa" en la naturaleza humana, de manera que esa ley es la que constituye a la naturaleza y no viceversa. El problema del número no ofrece dificultad alguna, cuando se trata del hombre, puesto que los números aparecen impresos en la razón humana del mismo modo que la Ley Eterna ${ }^{6}$. Cuando digo que tres y siete son diez, no puedo concebir que en otra parte o en otro tiempo sean nueve o sean once. Y lo que me acontece a mí acontece a todos los demás hombres: es una ley del pensamiento humano, una ley que constituye al pensamiento humano.

Dejando aparte el número en cuanto "manifestación" o revelación del ser, es decir, en cuanto forma o belleza, etc., veamos su sentido y valor ontológicos. Agustín reduce el número a la unidad y considera ésta como un elemento a priori de la razón humana; es pues claro que los números son leyes apriorísticas, puesto que todo número es una simple repetición de la unidad. Nadie puede conocer la unidad por experiencia, ya que toda experiencia es multiplicidad y nunca unidad ${ }^{7}$. El número ha de ser considerado como un valor y por lo mismo no puede darse sin un sujeto o razón que lo perciba como tal. Esto no significa subjetivismo puro. Las leyes de los números son leyes de nuestro pensamiento, ya que podemos aplicarlas a cantidades que jamás hemos soñado, en la seguridad de no equivocarnos ${ }^{8}$; pero no se trata de un capricho, sino de una "impresión", esto es, de una copia o imagen transcrita desde una zona lógica y objetiva. Así, concluye Agustín:

"La razón y la verdad de los números no pertenece al sentido corporal, sino que se mantienen inmutables y auténticas, y pueden contemplarlas en común todos los que raciocinan. Hay muchas otras cosas que son comunes, públicas y presentes a los que raciocinan, que permanecen intactas e incontaminadas ante los hombres: pero me sorprende que hayas citado esta razón y verdad del número... Porque no en vano los Santos Libros reúnen

6 Tracta... Impressa... Variantur... (Ibid.).

7 Numeros impressos esse animo nostro (Ibid. II, 8, 20, PL. 32, 1251. Ibid. II, 8, 22, PL. 32, 1252).

8 Ibid. 
la Sabiduría con el número, cuando dicen: "para que yo supiera, meditara y buscara la sabiduría y el número" (Eccle. 7,26$) 9$.

Cuatro puntos afirma aquí Agustín: a) el número constituye un reino objetivo, público, común, que no pertenece a la individualidad existencial de cada hombre ni a sus experiencias individuales; b) Es accesible o presente a todas las inteligencias individuales, por el mero hecho de ser inteligencias, sin necesidad de experiencia o existencia alguna concreta; c) Es apriorístico; d) Es equiparado a la sabiduría humana.

Por lo dicho, el número es conocido en la experiencia y con la experiencia. Por sí mismo es "impreso", no "expreso", y sólo en su expresión puede ser conocido, como una cinta fotográfica sólo puede ser reconocida una vez revelada. El número, en cuanto impreso, es un misterio óntico, la raíz misma del ser. Esta raíz o número es ya una base fija, estable, inmutable, desde la cual cobra sentido y valor el dinamismo, el movimiento, el proceso o evolución hacia la unidad ideal, la entelequia: es pues imposible imaginar en Agustín un dinamismo o actualismo puros. Pero es también imposible imaginar en él un estatismo o quietismo aristotélico. Habrá que pensar en una situación estadodinámica.

Es muy interesante para nosotros la identidad de número y sabiduría, que Agustín establece en el texto que estamos comentando y en los sucesivos, ya que acerca de la sabiduría nos ha hablado muchas veces y con claridad. Podremos aplicar así lo que nos dice acerca de la sabiduría profunda u originaria del hombre, al número de todas las cosas. Una vez establecida esa relación de identidad, de origen y de función, tenemos ya un modo claro de definir la naturaleza del número agustiniano. Poco importa para el caso la apelación a un texto bíblico, para establecer una doctrina filosófica. Parece claro que Agustín ha tomado ese texto bíblico de San Ambrosio ${ }^{10}$. En realidad, Agustín toma el texto como pretexto para confirmar una doctrina filosófica, ya establecida dentro de su platonismo.

El mismo Agustín se encarga de precisar cómo hemos de representarnos esa sabiduría que nos servirá de punto de comparación para definir el número. No hay múltiples sabidurías, una para cada individuo. La sabiduría es única para todos, objetiva, pública, común, accesible. Ser más o menos "sabios" es participar más o menos de ella. Esto significa,

9 Ibid. II, 8, 24, PL. 32, 1253. Ibid. II, 8, 21, PL. 32, 1251 s.

10 De Bono Mortis 7. 
por de pronto, que se trata sólo de una participación ${ }^{11}$. Ùn sujeto, por sí mismo, es pura potencialidad, puede "participar" del objeto, "objetivarse" en cierto modo, informarse, formarse o conformarse con un reino objetivo, lógico, eterno. ¿Qué es entonces la sabiduría?

\footnotetext{
"¿Piensas que la sabiduría es otra cosa que la verdad, en la que se contempla y posee el sumo bien?... Consta que todos queremos ser felices; y del mismo modo consta que queremos ser sabios, pues nadie puede ser feliz sin ser sabio. En efecto, nadie es feliz, sino en virtud de ese "sumo bien", que es contemplado y poseído en esa sabiduría que llamamos la verdad. Antes de ser felices, llevamos impresa en nuestra mente la noción de felicidad; gracias a esa noción, sabemos y afirmamos sin vacilación que queremos ser felices. Pues del mismo modo antes de ser sabios llevamos impresa en la mente la noción de sabiduría; gracias a esa noción, si alguien nos pregunta si queremos ser sabios, respondemos afirmativamente sin sombra alguna de duda" 12 .
}

La verdad o sabiduría es pues una ley impresa en la mente humana, ı'na aptitud o idoneidad, que permite al hombre descubrir y contemplar directamente una zona objetiva y lógica, que llamamos "la verdad". El objetivismo aristotélico queda así compensado en Agustín por un subjetivismo platónico y por otro subjetivismo cristiano más profundo. El existencialismo objetivista exige que cada individuo tenga su propia sabiduría y su propia verdad: cada individuo es una especie propia e irreductible, ya que por muy abierto que el individuo esté al mundo, es hijo de sus obras y de sus experiencias, siempre diferentes de las obras y experiencias de su vecino y aun de su hermano gemelo. Por eso Agustín refuerza sobre todo el subjetivismo, el aspecto formal del conocier, la luz a cuya influencia conocemos los abjetos diferentes.

"Son múltiples y diferentes las cosas, que los hombres contempilan a la luz del sol, pero es única la luz con que cada individuo ve y capta los objetos... Del mismo modo, son muchos y diferentes los bienes, entre los que cada individuo elige a su talante... Pero quizá esa luz de la sabiduría, por la que los hombres descubren y cobran tales bienes, sea única y común para todos los sabios" 13 .

¿Cómo es posible que un objeto sea común, si cada uno lo percibe según sus propias leyes y propiedades? Sin embargo, contra los hechos

11 De Libero Arbitrio II, 9, 25, PL. 32, 1254: Unam praesto esse communiter omnibus, cujus quanto magis quisque fit particeps, tanto est sapientior.

12 Ibid. II, 9, 26, PL. 32, 1254 s.

13 Ibid. II, 9, 27, PL. 32, 1255. 
no valen argumentos. Ahí tenemos, dice Agustín, los "primeros principios". Todos los ven con tal evidencia que sería ridículo recurrir a la experiencia individual, diferente en cada sujeto, para explicar la coincidencia total en aceptar esos principios. Se trata pues de algo que, aun sin dejar de ser "subjetivo", es realmente objetivo. Por eso se habla con toda exactitud y precisión de "impresión" o carácter. Esa impresión es el constitutivo radical: la llamada racionalidad consiste en ese número con que "juzgamos", es decir, con que comparamos lo empírico con lo eterno:

"No te pregunto más. Me basta saber que ves conmigo: concedes que son evidentes éstas como reglas o luz de las virtudes, que son verdaderas e inmutables, y que una por una o en conjunto están presentes en común para que las contemplen todos aquellos que puedan hacerlo cada uno con su propia mente o razón... Es pues evidente que todas estas que hemos llamado reglas o luminares de las virtudes, pertenecen a la sabiduría: en efecto, cuanto más y mejor las utiliza el individuo para organizar la propia vida, cuanto más se ajusta la vida a esas reglas, tanto más y mejor vive y obra con sabiduría... Luego, así como son verdaderas e inmutables las reglas de los números, accesibles a todos, así son verdaderas e inmutables las reglas (principios) de la sabiduría..." 14.

El paralelismo entre el número y la sabiduría lleva a Agustín a una conclusión previsible. ¿No se tratará de la misma realidad? Quizá el número y la sabiduría son la misma cosa, o quizá una realidad se derive de la otra, o quizá la una sea el constitutivo de la otra. Agustín atestigua que, cuando se pone a meditar en los principios de las matemáticas o en los de la sabiduría, se siente arrastrado a regiones remotas, alejadas de todo lo corporal. Y piensa que es necesario recurrir a la metáfora, para poderse explicar. Hay pues una región espiritual, que puede llamarse cubile, penetrale, regio, habitaculum, sedes numerorum, y que no es otra cosa que la "verdad" ${ }^{15}$. Esa metáfora, que responde en realidad a la regio longinqua de los platónicos, le lleva a pensar que se trata del "mundo inteligible".

Pero un nuevo texto bíblico le ofrece otro pretexto para continuar su meditación. Se trata de un texto importante, que será luego utilizado con frecuencia: "attingit a fine usque ad finem fortiter, et disponit omnia suaviter" (Sap. 8, 4). En este texto se enuncian dos cosas, y Agustín

14 Ibid. II, 10, 29, PL. 32, $1256 \mathrm{~s}$.

15 Cum haec duo sint in secretissima certissimaque veritate (Ibid. II, 11, 30, PL. 32, 1257). 
las aplica al tema que estamos estudiando. Cree que la primera parte del texto se refiere al número, mientras que la segunda parte se refiere a la sabiduría. El número y la sabiduría son pues "instrumentos", órganos, por los que el Creador llega a regir su creación ya con fortaleza (número), ya con suavidad (sabiduría). "De un fin a otro" parece significar concrétamente de una unidad' a otra, esto es, de la unidad radical de cada cosa hasta su unidad ideal o perfección. El número y la sabiduría son pues el órgano intermedio por el que el Creador lleva a cada ser desde la unidad, que sirve de punto de partida, hasta la unidad, que es el término o meta de llegada. Pero es un órgano o instrumento todavía misterioso, no expreso, oculto; utilizará luego otros géneros secundarios (verdad, amor) y se hará visible y cognoscible sólo por la experiencia, sólo por sus efectos. El número es pues otra vez virtus et sapientia, es decir, sujeto de conocimiento y de eros, entelequia radical, o razón y fundamento de toda entelequia, un "instinto de ser".

¿Cuál es la diferencia entre el número y la sabiduría? Se trata sin duda de dos conceptos diferentes y de dos vocablos que suenan de un modo diferente en los oídos y en la consciencia de los hombres. La diferencia consiste en que la sabiduría es "propia" del hombre, exclusivamente, mientras que el número es "propio" de todas las cosas, incluído también el hombre. Los cuerpos tienen números; las almas de los brutos tienen también números; en cambio, sola el alma racional, el alma humana, es sujeto de sabiduría. Dios ha distribuído los números a todas sus criaturas, pero el número de la razón humana se llama "sabiduría" o número racional. De ese modo, la razón "juzga" todos esos números inferiores o "irracionales" que van "impresos" en cada uno de los seres inferiores ${ }^{16}$. La diferencia es pues específica, dentro de un género común. La "razón" es el número radical del ser humano: le sirve al hombre para ser, es decir, para ser racional, y no sólo para pensar.

Agustín se vale de una nueva metáfora para explicarnos la coincidencia genérica entre el número y la sabiduría respecto a su fuente u origen, que es Dios. Compara el número y la sabiduría, que vienen de Dios, al "fulgor y calor" que vienen del fuego:

"En un fuego único sentimos como consustanciales el fulgor y el calor, sin que sea posible separarlos. El calor alcanza sólo a los objetos cercanos,

16 Ibid. II, 11, 31, PL. 32, 1258. El número y la sabiduría desempeñan pues la misma función de constituir el fundamento del ser. Pero esa función es superior en el hombre, ya que el ser racional y el ser irracional son diferentes. 
mientras que el fulgor llega mucho más lejos. Del mismo modo la potencia de entender, que radica en la inteligencia, pone en movimiento a los sujetos más cercanos, que son las almas racionales; en cambio, a los objetos más lejanos, que son los cuerpos (y las almas de los brutos) no los alcanza el calor de la sabiduría, sino sólo el fulgor de los números" 17.

Un tercer texto bíblico vuelve a servir a Agustín de pretexto para detenerse en el segundo sentido del término "número", es decir, en aquel sentido de forma, de expresión, de sustancia estética, consciente y científica, aplicable a la Ontología, Noética, Moral y Estética. "En los caminos se mostrará a ellos alegremente, y en toda providencia les saldrá al encuentro" (Sap. 6, I7). Aquí se trata ya de otra cosa, esto es, de la relación entre el número objetivo de las cosas y la razón subjetiva; dicho de otro modo, se trata de la inteligibilidad de los números. El número radical, en el primer sentido, de que antes hemos tratado, obliga al ser a ser, y por ende pone en movimiento todas sus potencialidades y propiedades. Los movimientos de ese ser, producidos por el número radical, se convierten a su vez en "números", para toda razón humana que quiera percibirlos. La "animadversio" platónica, de la que tanta veces habla Agustín, es un "signo" o "nutus", un carácter sacramental, que podemos percibir en todo lo visible:

"A donde quiera que te vuelvas, te habla (la Sabiduría divina) mediante cientos vestigios que imprimió en sus obras; cuando recaes en el mundo de las exterioridades, te vuelve a llamar para que te interioxices, mediante esas mismas formas de los objetos exteriores... Todo es numeroso... Pero no podrías aprobar o reprobar, si no poseyeras en tu interior la ley de la hermosura, con la que comparas toda hermosura sensorial" 18.

El número es por consiguiente la raíz estadodinámica de todo ser, órgano de una Potencia creadora, "tirante ontológico", que une al Creador con la Criatura.

Aprovecha nuevamente Agustín un texto bíblico, como pretexto, para explicar la relación de cad'a objeto con el acto divino creador, que imprime los números en las cosas: "Permaneciendo en sí misma ( La Sapientia) renueva todias las cosas" Sap. 7, 27). El "Uni-verso" de Agustín está en perpetuo movimiento renovador gracias a esa divina "Providencia", de que antes hablaba, que ha impreso los números en los objetos

17 Ibid. II, 11, 32, PL. 32, 1258.

18 Ibid. II, 16, 41, PL. 32, 1263. Cfr. De Ordine I, 8, 25 s., PL. 32, 989 s. 
como una representación activa, órgano o instrumento radical de Dios:

“Todas las cosas que existen, volverían a la nada, si perdieran enteramente su forma; porque la Forma inmutable, gracias a la cual subsisten las cosas mudables, para que vayan llenándose de los números propios de su forma, y sean movidas por ellos, es la providencia de tales cosas "19.

Es pues claro que en Agustín aparece una dialéctica radical, apoyada en su concepto del número, según nos dice en otra parte:

"Algunas cosas se cambian en mejores y por ende tienden a ser: tal mutación no se llama perversión o reversión, sino conversión. Porque la pervensión se opone al orden; en cambio, las cosas que tienden al ser tienden al orden; al conseguir el orden, consiguen el ser, en cuanto eso es posible para una criatura. En efecto, el orden organiza al objeto en una cierta conveniencia. $Y$ el ser no es otra cosa que ser uno. Por lo mismo una cosa en tanto es en cuanto consigue su interior unidad. La conveniencia o concordia es obra de la unidad, y por esta son, en cuanto son, aquellas cosas que implican composición. Las cosas simples son pox sí mismas, ya que no ocupan lugar; en cambio, las que no son simples, sólo pueden imitar la unidad con una concordia de partes, y en tanto son en cuanto consiguen imitarla." 20.

El pensamiento es pues claro. Hay una unidad fontanal, misteriosa, que es conjuntamente ejemplar o formal y virtual o enérgica, a la que se referirá Agustín con frecuencia para hacer múltiples aplicaciones:

"Dijo Dios: congréguese el agua que está bajo el cielo, es decir, organícese la materia corporal en una forma, para que sea ya este agua que sentimos. En una sola congregación. La misma potencia de la forma nos es presentada con el nombre de unidad. Porque lo que llamamos forma es organizar algo en unidad: en efecto, el Principio de toda forma es sumamente uno" 21 .

El número, la unidad radical, es pues potencia trófica, unificante, dentro del tiempo y del espacio, si se trata de cosas corporales o materiales. Mientras lo simple es per se unum, lo compuesto es unidad compuesta, correlación ad intra y ad extra, imitación de la unidad a su propio modo. Unificar es imprimir una forma, formar, sacar de la confusión, dar distinción, especie visible, perfección propia. Agustín piensa

19 Ibid. II, 17, 45, PL. 32, 1266.

20 De Mor. Eccl. II, 6, 8, PL. 32, 1348.

21 De Gen, ad litt. imp. liber 10, 32, PL. 34, 233. 
en una "mediación", como si no conviniese que Dios moviese la materia directamente, sino que la moviese mediante alguna causa segunda, mediate este instrumento $\mathrm{u}$ órgano que es el número o unidad fontanal. Dios imprime pues el número en los objetos irracionales y la sabiduría en los racionales.

En todos los seres, como última razón, se descubre un apetito de unidad, y ese apetito nos remite ya inmediatamente a Dios, unidad simplicísima y perfectísima:

"Todas las cosas que apetecen la unidad, tienen esa regla, forma, modelo (la Verdad-el Verbo), pues sola ella ha realizado la semejanza de Aquel que le dio el sex" 22 .

El apetito de unidad, el número radical, es pues imitación de Dios. Todo ente tiende a su propia unidad ideal, unidad perfecta, a su Idea divina, y esa es su ley natural, por la cual cada ser es su propia ley ${ }^{23}$. Tal es la ley fundamental de la Ontología, así como la ley de la verdad es el fundamento de la Noética, o la ley del amor es el fundamento de la Etica :

"Dice a los cuerpos: Si no estuvieseis retenidos en una unidad, no seríais nada; y si fueseis la misma unidad, no seríais cuerpos. Pero se le replica: ¿Por dónde conoces esa unidad, según la cual juzgas los cuerpos? Si no la conocieseis, no podriais juzgar que los cuerpos no la realizan plenamente... Luego la ves con la inteligencia. ¿Pero, dónde la ves?... Todo cuerpo es verdadero cuerpo, pero falsa unidad. Porque no es sumamente uno, ni imita la unidad tan perfectamente que la realice; pero no sería tampoco un cuerpo, si no fuese uno de algún modo. Como tampoco podría ser uno de algún modo, si no recibiera esa capacidad de Aquel que es sumamente uno" 24 .

Convendrá quizá repetir, para terminar, que el número agustiniano es misterioso, es el ser, es la raíz, es la unidad. No se diga pues que lo más profundo de las cosas es su verdad o es su amor. Tampoco en el hombre son la verdad o el amor lo más profundo, ya que la sabiduría, a que nos hemos referido es inconsciente, ontológica, y no pertenece a la Noética de por sí. No se diga tampoco que la fuerza elemental de las cosas o de los hombres es "la ley de la felicidad" o la "ley de la verdad".

22. De Vera Relig. 31, 58, PL. 34, 148.

23. Lex psa etiam ipse fit (Ibid.).

24. Ibid. 32, 60 y 34, 63, PL. 34, 149 s. 
Todo esto es ya consecutivo. La realidad o intención más profunda de las cosas y de los hombres es su número, su sabiduría radical. Y la ley o energía más profunda de la Ontología, orgánica o inorgánica, es el "apetito de unidad". Como se ve, Agustín, influído quizá por las especulaciones de Plotino, ha aceptado las bases neoplatónicas para organizar una dialéctica cristiana trinitaria. Agustín no llega, como Plotino, a establecer el "unum in nobis", o el "unum in rebus" como testimonio de una emanación a partir de Lo Uno. En efecto, en el Cristianismo hay que contar con una creación y no con una emanación. Pero, una vez cambiada la clave, es todavía posible pensar que lo que impera en el "Uni" "verso" es la "unidad", el número.

Nota.-¿Cómo se ha de relacionar el número con el modo? En el libro De Beata Vita toma Agustín el concepto de modo de Cicerón y de lós estoicos: el modo es el justo medio entre dos extremos viciosos ( $D e$ Beata Vita 4, 32, PL. 32, 975). Pero ya se introduce el concepto matizado por Plotino: lo Uno, el Bien, es "medida y límite de todas las cosas" (Plotino, Enn. I, 8, 2, 5). Lo Uno es mensura no mensurada (Enn. V, 5, 4). Por el contrario, el mal es carencia de medida y de orden, ausencia de forma (Enn. I, 8, 4). Pero Agustín entiende las cosas a su propio modo, como siempre. Para él, El Uno es Medida, mientras que la Verdad es Forma. El Uno mensura y limita a la Forma; el Uno es Medida universal y, en cuanto tal, carece de medida que lo mensure a El.

El famoso texto Sap. II, 21 : omnia in mensura et numero et pondere dispostisti, que Agustín convertirá luego en fundamento de su dialéctica trinitaria, es sólo un pretexto: ya había organizado la dialéctıca, cuando descubrió ese texto, que le venía muy bien para su propósito. Modo y Especie son dos principios o constitutivos del ser. Pero la cspecie (belleza, idea) se identifica y viene detrás del modo o número, con la forma. El Modo, al identificarse con el número, es mensura, límite, definición de la forma o esencia. De ese modo el concepto de "superhombre", por ejemplo, es un absurdo total, una contradicción radical: nada es superior a sí mismo; aunque el hombre llegase a ser un dios, sería simplemente un hombre, jamás un superhombre o "supercosa". Una esencia no puede ser nunca un ápeiron aunque evolucione indefinidamente.

Hay que tener en cuenta que Agustín, para acomodarse al texto kíblico, antes mencionado, identificó el modo ciceroniano y estoico con 
la mensura, aplicándola al Hijo y no al Padre. De ese modo, el modo, que inicialmente se identificaba con el número, se identifica luego con la mensura, esencia, forma o especie. Así queda ya firmemente establecido el término dialéctico: (numerus) modus-species-ordo. Véase, a modo de ejemplo, este texto en que Agustín todavía vacila en su terminología: omne quod est, aut est corporeum aut incorpereum. Corporeum sensibili, incorporeum autem intelligibili specie continetur. Omne igitur quod est, sine aliqua specie non est. Ubi autem aliqua species, necessario est aliquis modus. Et modus aliquid boni est. Summum ergo malum nullum modum habet; caret enim omni bonio. Non est igitur: quia nulla specie continetur, totumque hoc nomen mali de speciei privatione repertum est. De Div. Q., q. 6, PL. 40, 13. El término mensura, por su parte, tiene dos significados: a) medida de los áridos, fanega, hemina, etc.; b) cantidad de grano que entra en una fanega, hemina, etc.

P. L. Cilleruelo, O. S. A. 